\title{
Kidney ventrally rotation technique in retroperitoneal robot-assisted partial nephrectomy for posterior hilar tumor: technical feasibility and preliminary results
}

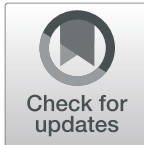

Xiaotao Yin $^{1+}$, Sinan Jiang ${ }^{1 \dagger}$, Zhiqiang Shao ${ }^{2 \dagger}$, Yongliang Lu', Jiaxiang Guo ${ }^{1}$, Yi Xiao ${ }^{1}$, Xiaoying Zhu', Hualiang Yu', Han Ma ${ }^{1}$, Yu Yang ${ }^{1}$ and Jiangping Gao ${ }^{1,3^{*}}$

\begin{abstract}
Purpose: The retroperitoneal robotic assisted partial nephrectomy (RAPN) is suitable for tumors locating on the posterior side of the kidney. However, the posterior hilar tumor poses an additional surgical challenge due to the special location and poor tumor exposure. We developed a novel kidney ventrally rotation technique to overcome this difficulty during retroperitoneal RAPN and evaluated its efficacy in a retrospective case-control comparative study.

Methods: From March 2016 to April 2019, a total of 39 patients with posterior renal hilar tumor underwent retroperitoneal RAPN. The kidney ventrally rotation technique, which improved the tumor exposure by opening the peritoneum and rotating the kidney ventrally, was applied in 24 cases, and the conventional RAPN was performed in the other 15 cases (control group). Perioperative data was analyzed to evaluate the efficacy of the kidney ventrally rotation technique.

Results: In kidney rotation group, the 24 patients underwent RAPN successfully without converting to open surgery or radical nephrectomy. The warm ischemia time was $17.4 \pm 6.6 \mathrm{~min}$, which was significantly shorter than $24.5 \pm$ $8.3 \mathrm{~min}$ in control group. The mean operation time $(80 \pm 24 \mathrm{~min})$ and estimated blood loss $(104 \pm 65 \mathrm{ml})$ were not different from the control group. No sever complications occurred, and no positive surgical margin was found in all the malignant cases. After 14 months follow-up, no recurrence or metastasis occurred in all cases.

Conclusion: Kidney ventrally rotation technique is safe and feasible for improving the exposure of posterior renal hilar tumor during retroperitoneal RAPN. It could be regarded as an efficient option for the management of posterior hilar tumor.
\end{abstract}

Keywords: Robotic surgery, Partial nephrectomy, Posterior hilar tumor, Retroperitoneal approach, Kidney rotation

\footnotetext{
*Correspondence: jpgao@163.com

${ }^{\dagger}$ Xiaotao Yin, Sinan Jiang and Zhiqiang Shao contributed equally to this work.

'Department of Urology, The Forth Medical Center of the PLA General Hospital, No. 51 Fucheng Rd, Haidian District, Beijing 100048, China

${ }^{3}$ Department of Urology, First Medical Center of the Chinese PLA General Hospital, Beijing, China

Full list of author information is available at the end of the article
}

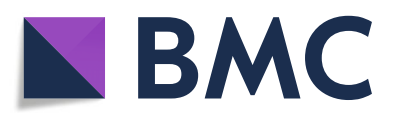

(- The Author(s). 2020 Open Access This article is licensed under a Creative Commons Attribution 4.0 International License, which permits use, sharing, adaptation, distribution and reproduction in any medium or format, as long as you give appropriate credit to the original author(s) and the source, provide a link to the Creative Commons licence, and indicate if changes were made. The images or other third party material in this article are included in the article's Creative Commons licence, unless indicated otherwise in a credit line to the material. If material is not included in the article's Creative Commons licence and your intended use is not permitted by statutory regulation or exceeds the permitted use, you will need to obtain permission directly from the copyright holder. To view a copy of this licence, visit http://creativecommons.org/licenses/by/4.0/. The Creative Commons Public Domain Dedication waiver (http://creativecommons.org/publicdomain/zero/1.0/) applies to the data made available in this article, unless otherwise stated in a credit line to the data. 


\section{Introduction}

Robot-assisted laparoscopic partial nephrectomy (RAPN) is demonstrated to be superior to conventional laparoscopic partial nephrectomy (LPN) in terms of estimated blood loss and warm ischemia time, because of the 3D vision and precise dissection of the robotic system $[1,2]$. Usually, the choice of operation approach during RAPN mainly depends on the tumor location, for example, transperitoneal approach for anterior tumor and retroperitoneal approach for posterior tumor [3]. The surgical management of posterior renal hilar tumor or posterior lip tumor is still a difficult challenge for urologists. The posterior hilar tumor locates behind the hilar vessels, so the retroperitoneal approach may be more direct and suitable without interference of hilar vessels. However, the exposure of posterior hilar tumor during retroperitoneal RAPN is still difficult in some cases because of the narrow retroperitoneal space, even for the experienced surgeon. Therefore, we propose a novel efficient kidney ventrally rotation technique, which improves the exposure of the posterior hilar tumor by opening the ventral peritoneum and rotating the kidney ventrally. In this article, we would describe this technique and evaluate its feasibility and efficacy in a retrospective casecontrol comparative study including patients who underwent retroperitoneal RAPN for posterior hilar tumor.

\section{Methods}

\section{Patients}

From March 2016 to April 2019, the patients with posterior hilar tumor $(\leq 7 \mathrm{~cm})$ that underwent retroperitoneal robotic-assisted partial nephrectomy were first screened. The posterior renal hilum tumor was defined as the tumor locating in the posterior renal hilum region and being close to the main renal vessels (Fig. 1). The cases that used a third robotic arm and instrument or a second hand-assisted trocar were excluded. Finally, 24 cases that were applied kidney ventrally rotation technique were included in the kidney rotation group, and 15 cases that underwent conventional retroperitoneal RAPN were included in control group. The preoperative computed tomography (CT) or magnetic resonance imaging (MRI) examination was evaluated to obtain the tumor parameters, including tumor size, tumor location, and and tumor complexity according to R.E.N.A.L nephrometry score [4]. All cases were diagnosed as single tumor on the posterior side of the renal hilum with no lymph node or renal vessel involvement. The metastatic cases were excluded by routine chest $\mathrm{x}$-ray or other specific scan according to clinical indication.

In our hospital, the patients were all informed that their clinical data might be used in future study without invasion of privacy during hospitalization. And this study was approved by the ethical committee of Forth Medical Center of the Chinese PLA General Hospital. The data of patients' demographic characteristics, tumor sizes, R.E.N.A.L scores, preoperative laboratory results, warm ischemic time, estimated blood loss, operation related complications, and pathologic results were collected retrospectively. And all the patients were followed postoperatively according to the recommendation of the EAU guideline [5].

\section{Operation procedure}

All operations were performed by the same surgeon with DaVinci Si surgical system. After adopting total anesthesia with tracheal intubation, the patients were positioned in the full-flank lateral decubitus position.
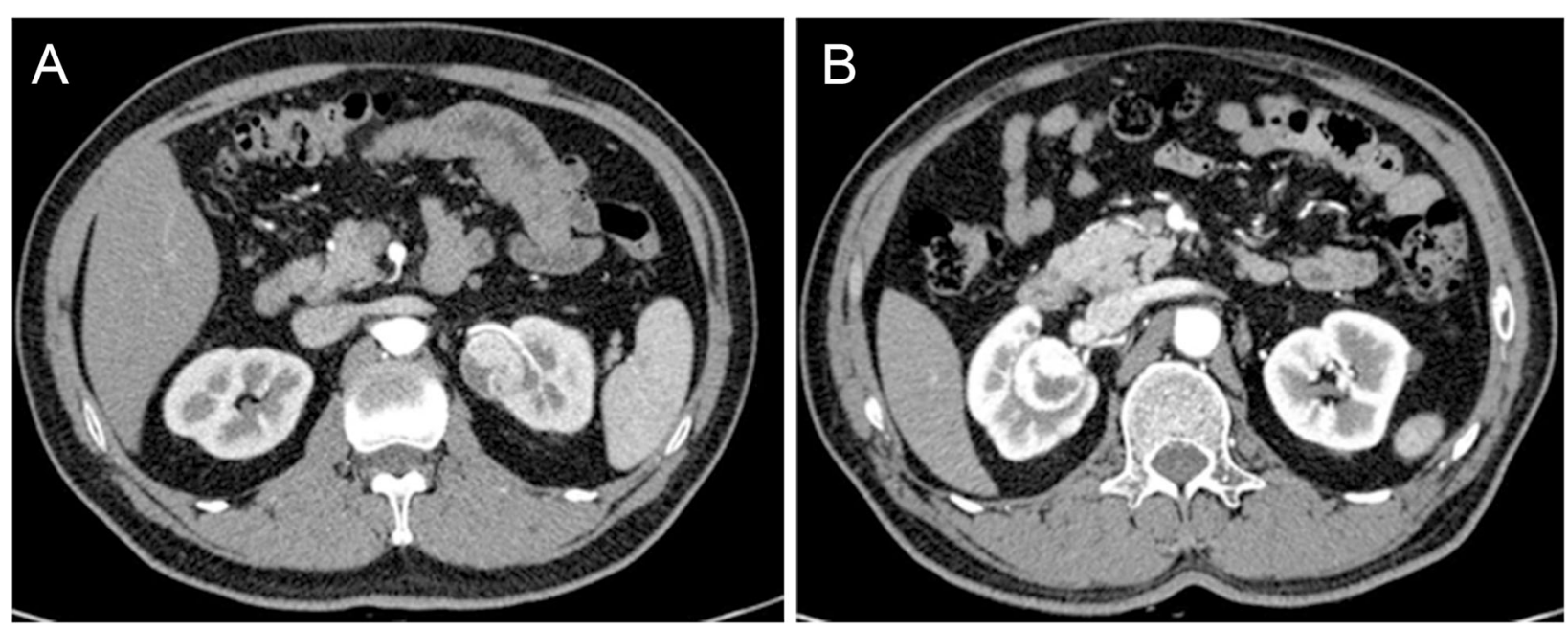

Fig. 1 a Renal-enhanced computed tomography scan showing posterior hilar tumor (left side). b Renal-enhanced computed tomography scan showing posterior hilar tumor (right side) 
Patients' affected sides were upward and vertical to the operation bed. The retroperitoneal space was established by modified method of finger dissection [6]. First, the skin and subcutaneous tissue at $2 \mathrm{~cm}$ above the iliac crest on the mid-axillary line were incised for $2 \mathrm{~cm}$. Then, the muscle and lumbodorsal fasciae were dissected with vessel forceps, and the retroperitoneal space was dissected bluntly with finger. A 12-mm trocar was placed at this site for camera. Under the guidance of the lens, two $8 \mathrm{~mm}$ trocars were placed about $2 \mathrm{~cm}$ above the plane of the camera trocar along the anterior and posterior axillary line respectively. Another $12 \mathrm{~mm}$ trocar was placed $2 \mathrm{~cm}$ above the superior spine as assistance port. The robot enters from the longitudinal direction of the patient's head side, then the machine docking was completed.

The perirenal fasciae were incised longitudinally after the extraperitoneal fat was removed. The renal artery and vein were dissected, and the posterior hilar tumor could not be exposed with satisfaction (Fig. 2a).

The conventional retroperitoneal RAPN was performed by direct tumor resection and kidney suture, while the kidney ventrally rotation technique was performed as follows.

\section{Kidney ventrally rotation procedure}

First, incise the peritoneum at the wrinkles or weakness (Toldt's line) (Fig. 2b), up to the hepatic flexure of colon for right kidney, even cut the triangular ligament, and the

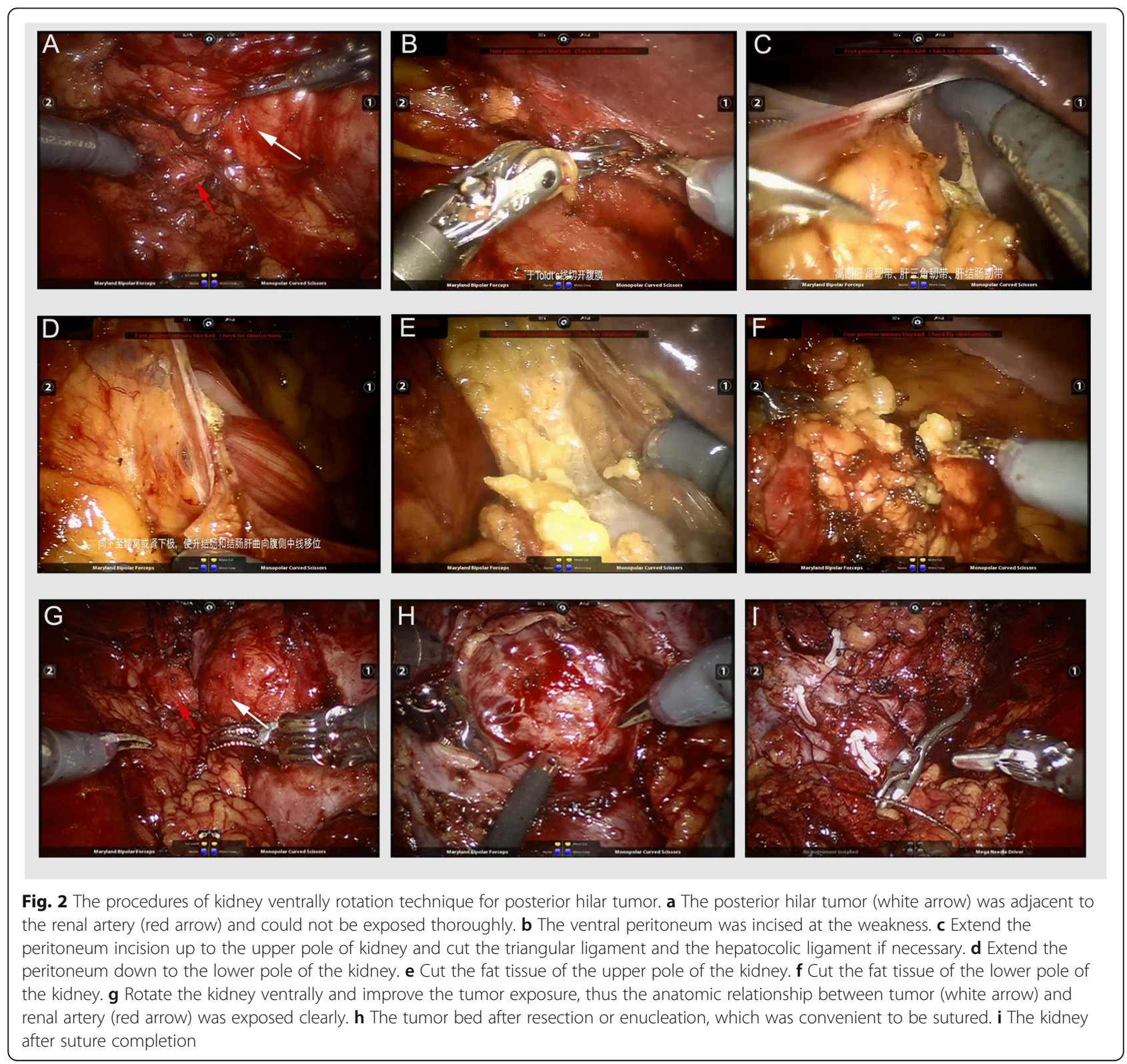


hepatocolic ligament if necessary (Fig. 2c). And for the left kidney, incise the peritoneum up to the spleen upper edge and cut the splenorenal ligament and splenocolic ligament if necessary. Then, the peritoneum was cut down to the lower edge of the kidney (Fig. 2d). The posterior side of the kidney was separated along the space between the renal parenchyma and the perirenal fat, and the adipose tissue of the upper (Fig. 2e) and lower (Fig. 2f) poles of the kidney is cut off respectively. The intraperitoneal bowel could drop down to the contralateral side due to the gravity on lateral position, and the kidney could be also ventrally rotated automatically or simply by retracting. Thus, the posterior hilar tumor could be exposed to the center of the surgical field without bowel interfering (Fig. 2g). The schematic of the kidney ventrally rotation technique was shown in Fig. 3. And the videos of kidney ventrally rotation technique were also uploaded on the website (Additional files).

Partial nephrectomy is performed after routine clamping of the renal artery (and, if necessary, the renal vein).
Stitching is performed using a knot-free barbed thread continuous method (Fig. 2h, i).

\section{Results}

Patients and tumors characteristics were summarized in Table 1 . The kidney rotation group included 18 males and 6 females, with mean age of $52.6 \pm 14.5$ years (range $26-$ 78 years). The mean body mass index was $25.67 \pm 3.94 \mathrm{~kg} /$ $\mathrm{m}^{2}$ (range $18.64-35.49 \mathrm{~kg} / \mathrm{m}^{2}$ ). The mean tumor size was $4.3 \pm 1.7 \mathrm{~cm}$ (range $2.5-8 \mathrm{~cm}$ ). The median R.E.N.A.L score was 9 (range 7-12). All the 24 patients underwent RAPN successfully without converting to open surgery or radical nephrectomy. The control group included 10 males and 5 females, with mean age of $62.5 \pm 9.4$ years, mean BMI of $24.64 \pm 4.21 \mathrm{~kg} / \mathrm{m}^{2}$, mean tumor size of 3.8 $\pm 1.6 \mathrm{~cm}$, and median R.E.N.A.L score of 8 .

The perioperative outcomes were listed in Table 2 . The warm ischemia time was $17.4 \pm 6.6 \mathrm{~min}$ in the kidney rotation group, which was significantly shorter than $24.5 \pm 8.3 \mathrm{~min}$ in the control group $(P<0.05)$. There
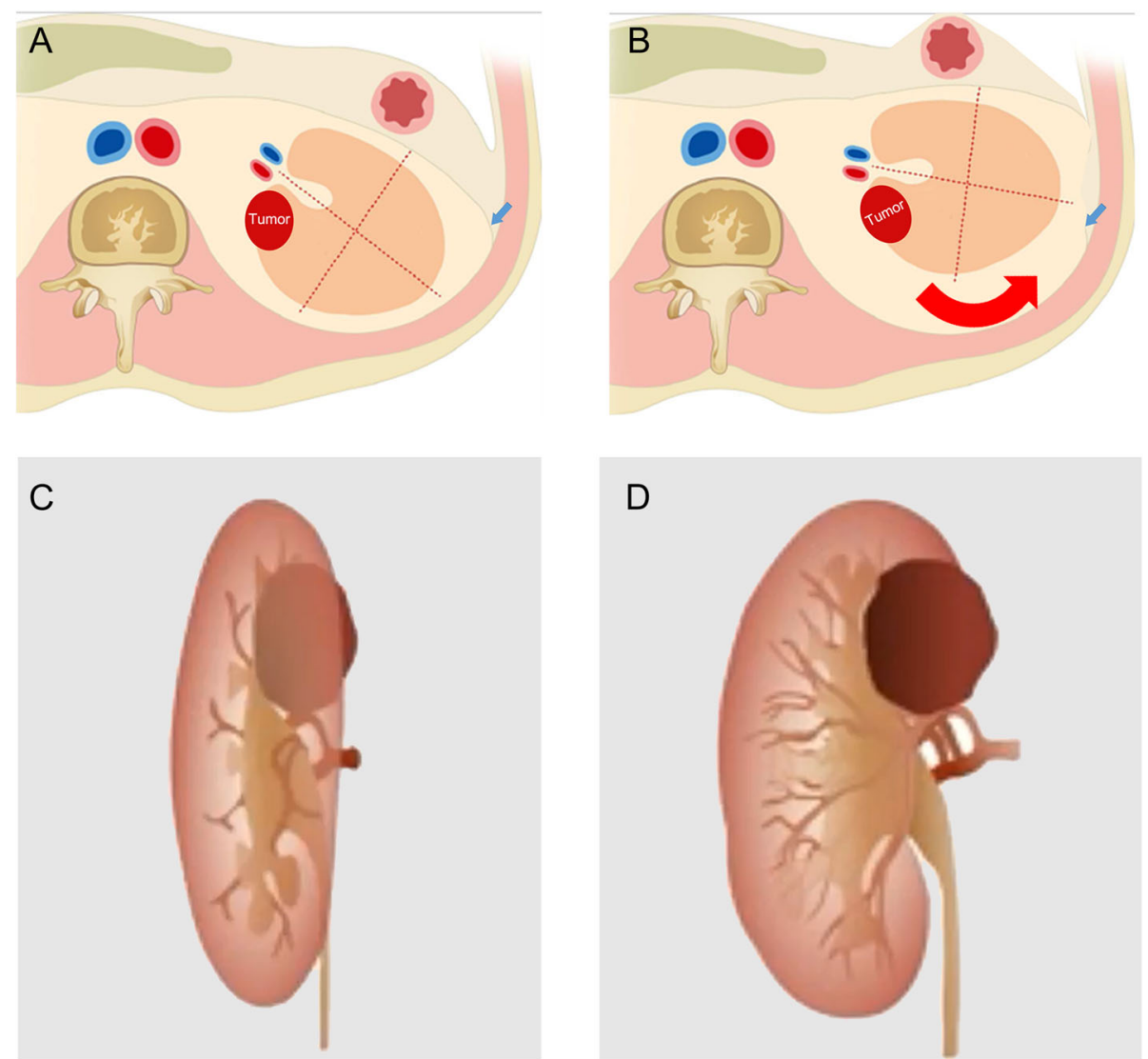

Fig. 3 The schematic of the kidney ventrally rotation technique. a The tumor located at the posterior side of the renal hilum, which was not at the center of the operation field during retroperitoneal RAPN. The wrinkles or weakness of the peritoneum was selected as the incising site (blue arrow). $\mathbf{b}$ The ventral peritoneum was extended cephalad and caudally, and the kidney was rotated ventrally. Consequently, the tumor exposure was improved enough for the further resection and suture. c The longitudinal view of the posterior hilar tumor during retroperitoneal RAPN. $\mathbf{d}$ The improved exposure of the tumor after kidney ventrally rotation 
Table 1 Demographic and tumor characteristics

\begin{tabular}{lll}
\hline Variables & Kidney rotation & Control \\
\hline Patients, $n$ & 24 & 15 \\
Age, yr, mean (SD) & $52.6(14.5)$ & $62.5(9.4)$ \\
Male gender, $n$ (\%) & $18(75.0)$ & $10(66.7)$ \\
BMI, mean (SD) & $25.67(3.94)$ & $24.64(4.21)$ \\
RENAL score, median (range) & $9(7-12)$ & $8(7-11)$ \\
Tumor size, cm, mean (SD) & $4.3(1.7)$ & $3.8(1.6)$ \\
\hline
\end{tabular}

$B M I$ body mass index, $\mathrm{CCl}$ Charlson comorbidity index, $S D$ standard deviation.

were no significant differences between the kidney rotation group and control group in operation time and the estimated blood loss $(P>0.05)$. In the kidney rotation group, no abdominal organ damage, vascular injury, or subsequent bleeding occurred. Pathological results showed 18 cases of clear cell carcinoma, 4 cases of angiomyolipoma, 1 case of papillary carcinoma, and 1 case of oncocytoma. No positive surgical margin was found in all cases. After the operation, all the patients were followed as the recommended schedule, and the median follow-up time was 14 months. No recurrence or metastasis occurred in all patients.

\section{Discussion}

Multiple studies have demonstrated a comparable cancer-specific survival for PN vs RN-treating pT1 RCC $[7,8]$. In addition, PN demonstrated better preserved kidney function, thereby potentially lowering the risk of development of cardiovascular disorders $[9,10]$. So, PN is increasingly becoming a preferred choice for surgeon and patients with confined renal tumor. Robotic surgical system could shorten the learning curve and warm ischemia time, because of its unique three-dimensional

Table 2 Perioperative outcomes

\begin{tabular}{llll}
\hline Variables & $\begin{array}{l}\text { Kidney rotation } \\
(n=24)\end{array}$ & $\begin{array}{l}\text { Control } \\
(n=15)\end{array}$ & $P$ \\
\hline WIT, min (SD) & $17.4(6.6)$ & $24.5(8.3)$ & $*$ \\
EBL, ml (SD) & $104(65)$ & $86(60)$ & - \\
Operation time, min (SD) & $80(24)$ & $83(30)$ & - \\
Operation conversion, $n$ (\%) & $0(0 \%)$ & $0(0 \%)$ & - \\
Positive surgical margin, $n(\%)$ & $0(0 \%)$ & $0(0 \%)$ & - \\
Median follow-up, mon (median) & 14 & 18 & - \\
Recurrence or metastasis, & $0(0 \%)$ & $0(0 \%)$ & - \\
$n$ (\%) & & & \\
Final pathology & & $12(80.0)$ & \\
$\quad$ Clear cell carcinoma, $n(\%)$ & $18(75.0)$ & $1(6.7)$ & \\
$\quad$ Papillary carcinoma, $n(\%)$ & $1(4.2)$ & $0(0)$ \\
$\quad$ Oncocytoma, $n$ (\%) & $1(4.2)$ & $2(13.3)$ & \\
$\quad$ Angiomyolipoma, $n(\%)$ & $4(16.7)$ &
\end{tabular}

WIT warm ischemia time, EBL estimated blood loss, SD standard deviation ${ }^{*} P<0.05,-P>0.05$ vision, precise operation, and flexible instruments. As for oncological outcomes, many studies have indicated that the RAPN has no significant differences with LPN or open PN, while RAPN is superior to $\mathrm{LN}$ and OPN in terms of estimated blood loss, warm ischemia time, hospital stay, and preserved effective nephron [11].

Like conventional LPN, RAPN usually has two conventional approaches: transperitoneal and retroperitoneal, depending on the location of the tumor [3]. Chinese urologists are more familiar with the retroperitoneal anatomy, as retroperitoneal LPN is predominant in most Chinese hospitals. Especially for tumors located on the posterior side of kidney, retroperitoneal approach is more suitable because of the direct access to tumor without the excess disturbance of the abdominal organs. And some studies have demonstrated that retroperitoneal approach has the advantages of patients' quicker recovery from operations and less postoperative complications.

Even with the application of robots, the surgical difficulty of renal hilar tumors is still significantly higher than that of non-hilar tumors $[12,13]$. The average operation time and renal ischemic time is longer, intraoperative blood loss is more, and the rate of intraoperative conversion to radical resection is higher for hilum tumor [14]. Posterior hilar or lip tumor poses additional technical challenges to the operating surgeon. Conventional transperitoneal approach may not be suitable despite of the large operation space, because the tumor is located behind hilar vessels, which interferes with the whole tumor dissection and renorrhaphy process. Retroperitoneal approach is more direct and appropriate for posterior hilar tumor, but the tumor exposure may be still unsatisfied in some cases which would hinder the tumor resection and suture processes. We also attempted to rotate the kidney ventrally without incising the peritoneum after dissociating the kidney completely from the fat layer during RARN, but the degree of rotation was limited and the improvement of tumor exposure was not satisfied. Some studies used the fourth mechanical arm on the ventral side for renal traction fixation during retroperitoneal RAPN, which could reduce the complications and margins caused by poor exposure and unclear vision [15]. However, this method consumed extra instrument or assistant, which increased the operation cost.

In this study, we freed the kidney by opening the peritoneum and rotated the kidney ventrally, which could maximally expose posterior hilar tumors. Among our 24 cases with posterior hilar tumor, the mean tumor size was $4.3 \pm 1.7 \mathrm{~cm}$, and the median R.E.N.A.L score was 9, which indicated the difficulty and complexity in these operations. Compared with the conventional method, the kidney ventrally rotation technique significantly improved the tumor exposure and reduced the difficulty of tumor resection and suture process, which achieved a 
shorter warm ischemia time $(17.4 \mathrm{~min}$ vs $24.5 \mathrm{~min}, P<$ $0.05)$. Besides, the kidney ventrally rotation process was not complicated or time-consuming. The whole operation time in the two groups was not significantly different $(P>.05)$. In the kidney rotation group, no case was converted to radical nephrectomy or open surgery, and no positive surgical margin or other postoperative major complications occurred. Moreover, this technique could also be adopted in conventional retroperitoneal LPN. In summary, although this technique attenuated the isolation character of retroperitoneal space, it facilitated the management of the posterior hilar tumor during retroperitoneal RAPN significantly, especially for surgeons without extensive surgical experience. We summarized four important steps which should be emphasized.

\section{Incising peritoneum}

The incision location of the peritoneum was selected in the weak or wrinkled place, such as the Toldt's line. Then, the incision must be extended cephalad and caudally along the paracolic sulcus under the direct vision. The incision extension could be adjusted during the whole operation depending on the tumor exposure. It was necessary to observe the abdominal organs during the incision process to avoid accidentally injuring, such as the intestine, liver, spleen, pancreas, and the diaphragm. The patient with history of abdominal surgery or intensive abdominal adhesion may not be suitable for this technique, because they had the increased risk of organ injury.

\section{Rotating the kidney}

As the kidney is fixed by the surrounding adipose tissue, simply cutting the peritoneum would not free the kidney adequately. Therefore, it is necessary to free the kidney by dissecting adipose tissue of upper and lower poles of the kidney, so that the kidney can be automatically rotated ventrally or simply by kidney retraction. As followed, the posterior hilar tumor would be turned towards surgical filed and fully exposed, which could decrease the surgical difficulty significantly.

\section{Resection}

The tumor resection is another technical challenge in partial nephrectomy for posterior hilar tumor. By kidney ventrally rotation method, the tumor exposure could be improved, which is the prerequisite for successful tumor resection. Because the tumor is close to renal vessels and collecting system, the accidental damage should be avoided during the resection process. The tumor enucleation technique could be used, which dissects the tumor mainly by blunt excision along tumor pseudocapsule. This technique does not increase the risk of positive margin, which has been reported in some other studies
[16]. If necessary, 3D reconstruction using enhanced CT or MRI scan data could also be applied to understand the tumor location, depth, and the relationship with blood vessels or collecting system.

\section{Renorrhaphy}

For the hilar tumor, the suture method for conventional tumor may be not suitable. The parenchyma edge of tumor bed after resection is close to renal sinus, which contains main branches of renal vessels and collecting system. Besides, the parenchyma edge close to renal sinus is not enough thick and may be cut through by the thread when performing the conventional kidney renorrhaphy. Therefore, we recommend the ring or c-shaped suture technique in the renorrhaphy of hilar tumor, which could reduce the difficulty of renorrhaphy and maximally preserve the effective nephron [17]. It is best to start stitching from the side close to renal sinus, which ensures that the blood vessel is not damaged as much as possible.

Several limits exist in this study, including small sample size and retrospective nature, while our study proposed a novel surgical technique for posterior hilar tumors and verified its feasibility, safety, and outcome in a preliminary group of cases. And prospective and controlled study with lager sample size is needed further.

\section{Conclusion}

Our study reports the preliminary results of kidney ventrally rotation technique in retroperitoneoscopic RAPN for posterior hilar tumor. The technique could effectively improve the exposure of posterior renal hilar tumor, consequently decreasing the difficulty and risk of the operation for tumors with this special location. The technique could be a useful and reasonable option for the management of posterior hilar tumor during retroperitoneal RAPN.

\section{Supplementary information}

Supplementary information accompanies this paper at https://doi.org/10 1186/s12957-020-01928-2.

Additional file 1. Incise the peritoneum to upper pole of the kidney. Additional file 2. Incise the peritoneum to lower pole of the kidney. Additional file 3. Cut the fat tissue of the upper pole of the kidney. Additional file 4. Cut the fat tissue of the lower pole of the kidney. Additional file $\mathbf{5}$. Rotate the kidney ventrally and block the renal vessels.

\section{Abbreviations}

PN: Partial nephrectomy; LPN: Laparascopic nephrectomy; RAPN: Roboticassisted partial nephrectomy; OPN: Open partial nephrectomy;

$\mathrm{CT}$ : Computerized tomography; MRI: Magnetic resonance imaging;

CCl: Charlson comorbidity index; BMl: Body mass index; EBL: Estimated blood loss; WIT: Warm ishemia time; SD: Standard deviation; PSM: Positive surgical margin; RCC: Renal cell carcinoma 


\section{Acknowledgements}

Not applicable.

\section{Consent to participate}

During hospitalization, patients were all informed that their clinical data might be used in future study without invasion of privacy.

\section{Authors' contributions}

Conception and design: X Yin, S Jiang, and J Gao. Acquisition of the data: $X$ Yin, S Jiang, Z Shao, Y Lu, and J Guo. Analysis and interpretation of the data: $H Y$ Yu, X Zhu, and $H$ Ma. Drafting of the article: X Yin, S Jiang, and Z Shao. Critical revision of the article: Y Xiao, Y Yang, and J Gao. All authors have viewed and approved the final version of the manuscript.

\section{Funding}

This work is granted by the National Key R\&D Program of China (Grant/ Award Number: 2017YFC0110800).

\section{Availability of data and materials}

The data that support the findings of this study are available from the corresponding author upon reasonable request.

\section{Ethics approval and consent to participate}

All procedures performed in studies involving human participants were in accordance with the ethical standards of the institutional and/or national research committee and with the 1964 Helsinki declaration and its later amendments or comparable ethical standards. This study was approved by the Ethics Committee of Chinese PLA General Hospital.

\section{Consent for publication}

Informed consent for publication was obtained from all authors.

\section{Competing interests}

None.

\section{Author details}

'Department of Urology, The Forth Medical Center of the PLA General Hospital, No. 51 Fucheng Rd, Haidian District, Beijing 100048, China. ${ }^{2}$ Department of Urology, Linyi People's Hospital, Linyi, China. ${ }^{3}$ Department of Urology, First Medical Center of the Chinese PLA General Hospital, Beijing, China.

Received: 1 April 2020 Accepted: 21 June 2020

Published online: 30 June 2020

\section{References}

1. Ludwig WW, Gorin MA, Pierorazio PM, Allaf ME. Frontiers in robot-assisted retroperitoneal oncological surgery. Nat Rev Urol. 2017;14(12):731-41.

2. Masson-Lecomte A, Yates DR, Hupertan V, Haertig A, Chartier-Kastler E, Bitker $\mathrm{MO}$, et al. A prospective comparison of the pathologic and surgical outcomes obtained after elective treatment of renal cell carcinoma by open or robot-assisted partial nephrectomy. Urol Oncol. 2013;31(6):924-9.

3. Haber GP, White WM, Crouzet S, White MA, Forest S, Autorino R, et al. Robotic versus laparoscopic partial nephrectomy: single-surgeon matched cohort study of 150 patients. Urology. 2010;76(3):754-8.

4. Kutikov A, Uzzo RG. The R.E.N.a.L. nephrometry score: a comprehensive standardized system for quantitating renal tumor size, location and depth. J Urol. 2009;182(3):844-53.

5. Capogrosso P, Capitanio U, La Croce G, et al. Follow-up after treatment for renal cell carcinoma: the evidence beyond the guidelines. Eur Urol Focus. 2016;1(3):272-81.

6. Gao J, Guo G, Jie Z, Wei W, Xu A, Dong J, et al. A fast method to identify renal vessels during retroperitoneal laparoscopic nephrectomy. J Endourol. 2008;22(8):1705-8.

7. Van Poppel H, Da Pozzo L, Albrecht W, Matveev V, Bono A, Borkowski A, et al. A prospective, randomised EORTC intergroup phase 3 study comparing the oncologic outcome of elective nephron-sparing surgery and radical nephrectomy for low-stage renal cell carcinoma. Eur Urol. 2011;59(4): 543-52.

8. Gratzke C, Seitz M, Bayrle F, Schlenker B, Bastian PJ, Haseke N, et al. Quality of life and perioperative outcomes after retroperitoneoscopic radical nephrectomy (RN), open RN and nephron-sparing surgery in patients with renal cell carcinoma. BJU Int. 2009;104(4):470-5.

9. Miller DC, Schonlau M, Litwin MS, Lai J, Saigal CS. Urologic diseases in America P. renal and cardiovascular morbidity after partial or radical nephrectomy. Cancer. 2008;112(3):511-20.

10. Thompson RH, Boorjian SA, Lohse CM, Leibovich BC, Kwon ED, Cheville JC, et al. Radical nephrectomy for pT1a renal masses may be associated with decreased overall survival compared with partial nephrectomy. J Urol. 2008; 179(2):468-71 discussion 72-3.

11. Peyronnet B, Seisen T, Oger E, Vaessen C, Grassano Y, Benoit T, et al. Comparison of 1800 robotic and open partial nephrectomies for renal tumors. Ann Surg Oncol. 2016;23(13):4277-83.

12. Dulabon LM, Kaouk JH, Haber GP, Berkman DS, Rogers CG, Petros F, et al. Multi-institutional analysis of robotic partial nephrectomy for hilar versus nonhilar lesions in 446 consecutive cases. Eur Urol. 2011:59(3):325-30.

13. Miyake H, Hinata N, Imai S, Furukawa J, Tanaka K, Fujisawa M. Partial nephrectomy for hilar tumors: comparison of conventional open and robotassisted approaches. Int J Clin Oncol. 2015;20(4):808-13.

14. Eyraud R, Long JA, Snow-Lisy D, Autorino R, Hillyer S, Klink J, et al. Robotassisted partial nephrectomy for hilar tumors: perioperative outcomes. Urology. 2013;81(6):1246-51.

15. Feliciano J, Stifelman M. Robotic retroperitoneal partial nephrectomy: a fourarm approach. JSLS. 2012;16(2):208-11.

16. Carini M, Minervini A, Masieri L, Lapini A, Serni S. Simple enucleation for the treatment of PT1a renal cell carcinoma: our 20-year experience. Eur Urol. 2006:50(6):1263-8 discussion 9-71.

17. Zhang C, Li X, Yu W, Zhang Q, Zhou L, He Z. Ring suture technique in retroperitoneal laparoscopic partial nephrectomy for hilar cancer: a new renorrhaphy technique. J Endourol. 2016;30(4):390-4.

\section{Publisher's Note}

Springer Nature remains neutral with regard to jurisdictional claims in published maps and institutional affiliations.

\section{Ready to submit your research? Choose BMC and benefit from:}

- fast, convenient online submission

- thorough peer review by experienced researchers in your field

- rapid publication on acceptance

- support for research data, including large and complex data types

- gold Open Access which fosters wider collaboration and increased citations

- maximum visibility for your research: over $100 \mathrm{M}$ website views per year

At $\mathrm{BMC}$, research is always in progress.

Learn more biomedcentral.com/submissions 